\title{
UN PROVINCIAL DANS L'ITALIE AUGUSTEENNE : UN AUTRE REGARD SUR LA «PATAVINITE » DE TITE-LIVE
}

\begin{abstract}
Summary: Livy was born in Padua, among the Venetes, in a part of Italy which received Roman citizenship only in $49 \mathrm{BC}$ and he was very proud of the origins of his little hometown - that could take pride in being, like Rome, a Trojan foundation. Indeed, before telling Aeneas' arrival on the shore of Latium, he begins his Roman history telling the story of Antenor, the Trojan hero who founded Padova. Later, he insists on the victory of his fellow-citizens in $302 \mathrm{BC}$ over the Spartan Cleonymos, one of the Greek generals who were appointed by the Tarentines to protect them from their enemies - a victory which appears to be a kind of anticipation of that of Rome against Pyrrhus. But Livy was well conscious that, in present times, the leading center in Italy and elsewhere was only Rome: the last time we hear of his native town in the extant books of his work is $174 \mathrm{BC}$, when the Romans had to restore order and peace in the Venetian town - an event which was considered so important in local memory that it was considered as the beginnig of a new era. The deep attachment of a provincial Roman like Livy to his little hometown did not prevent from feeling himself a member of the larger Roman comunity and resenting a strong attachment to Rome, head of the whole oikoumene and common patria of all Italians. He gives us a good example of the construction of a Roman Italy under Augustus.
\end{abstract}

Key words: Antenor, Cleonymos, Aeneas, Galli, Italia, patauinitas, patria, Pyrrhus, Roma, Tarentum, Livy, Troy, Trojans, Venetes, Virgil

La patauinitas qu'aux dires de Quintilien, Asinius Pollion reprochait à Tite-Live ${ }^{1}$, a fait couler beaucoup d'encre. Déjà en 1751, dans l'article " patavinité », à la page 162 du tome XII de la grande entreprise de la philosophie du XVIII ${ }^{\mathrm{e}}$ siècle que fut l'Encyclopédie ou dictionnaire raisonné des sciences, des arts et des métiers dont il avait été le coordinateur et l'animateur avec Diderot, le philosophe d'Alembert écrivait : « Les critiques se sont donné des peines infinies pour découvrir en quoi consistoit cette patavinité ». Il avait beau jeu de passer en revue toutes les interprétations qui avaient été données de son temps - explication politique, par le « pompéianisme »

${ }^{1}$ Quintilien, Institution oratoire 1.5. 56 (Pollio reprendit in Liuio patauinitatem) et 8.1 .3 (in Tito Linio, mirae facundiae uiro, putat inesse Pollio Asinius quandam patauinitatem). 
de l'historien², selon Paolo Beni (1552-1625), qui fut à la fin de sa vie professeur à Padoue, une orthographe déficiente, selon Lorenzo Pignoria (1571-1631), qui était padouan, une mauvaise prononciation, selon un Français, le père Rapin (1621-1687), une question de style, selon l'Allemand Daniel Georg Morhof (1639-1691), la présence de provincialismes linguistiques, selon un des plus célèbres professeurs à la Sorbonne, Charles Rollin (1661-1741) - pour conclure avec ironie sur le fait que $\ll \mathrm{M} . \mathrm{Balzac}^{3}$ ne pouvoit pas mieux rendre son radoteur ridicule, qu'en supposant qu'il se glorifioit d'avoir découvert ce que c'étoit que la patavinité reprochée à Tite-Live par Pollion ». Le débat se poursuit de nos jours, mais il n'y a pas lieu de reprendre ici cet aspect, toujours problématique, du rapport du grand historien de Rome avec sa cité natale ${ }^{4}$. En tout cas, l'hostilité que suscitait ainsi chez Asinius Pollion - qui était pourtant lui aussi un provincial, puisque sa famille était d'origine marrucine - la permanence du caractère padouan de l'auteur des $A b$ Vrbe condita libri montre la force du lien qu'il conservait avec sa ville natale, lien qui était assez fort pour qu'il la choisît comme lieu de sa dernière demeure 5 . Si bien que cette "patavinité » de Tite-Live, loin de toute utilisation polémique et donc par-delà le sens que le créateur du mot avait voulu lui donner, peut être aussi lue comme le signe de ce qu'un représentant aussi éminent des lettres romaines, fixé depuis longtemps dans l'Vrbs et ayant des liens personnels avec le prince ${ }^{6}$, gardait un attachement profond avec une ville qui ne

${ }^{2}$ On connaît le reproche que, selon Tacite, Auguste avait adressé à Tite-Live, Annales 4. 34, à cause de l'éloge qu'il avait fait de Pompée (Titus Liuius, eloquentiae ac fidei praeclarus in primis, Cn. Pompeium tantis laudibus tulit ut Pompeianum eum Augustus appellaret; neque id amicitiae eorum offecit).

${ }^{3}$ Il s'agit ici de l'écrivain, conseiller du roi et académicien Jean-Louis Guez de Balzac, né à Angoulême en 1597 et mort en 1654 .

${ }^{4}$ Nous nous bornerons à citer l'article de FLOBERT, P. : La patauinitas de Tite-Live d'après les mœurs littéraires du temps. Revue des Études Latines 59 (1981) 193-206 (définissant cette notion comme un manque d'élégance de style, d'urbanitas) ; mais, signe de la persistance du débat, J. N. ADAMS (The Regional Diversification of Latin (200 BC-AD 600). Cambridge 2007, 152-153) refuse l'idée que le terme, comme le voulait notre compatriote, s'applique à la macrologia que Quintilien reprochait à l'historien.

${ }^{5}$ Tite-Live est né soit en 59, soit en 64 av. J.-C. : la chronique de Jérôme, pour son année 1958, le fait naître à la première date, sous le consulat de César et Bibulus, mais comme elle précise que Valerius Messala Corvinus était né cette même année (Messala Coruinus orator nascitur et T. Liuius Patauinus scriptor historicus), il peut y avoir eu une confusion avec l'année 64, où les consuls furent César et Figulus). Il mourut à Padoue en 17, comme le précise Jérôme pour l'année 2033 (Liuius historiographicus Pataui moritur). Nous possédons vraisemblablement l'épitaphe de l'historien; ce doit être l'inscription $C I L$ V 2975, correspondant à la tombe familiale où il reposait avec son épouse Cassia Prima et ses deux fils T. Livius Priscus et T. Livius Longus (T. Liuius C. f. sibi et suis T. Liuio T. f. Prisco f. T. Liuio T. f. Longo f. Cassiae Sex. Primae uxori).

${ }^{6}$ Nous n'entrerons pas ici dans le débat sur les relations entre Tite-Live et Auguste (marqué par des études reprenant perfois les mêmes titres comme de DESSAU, H. : Livius und Augustus. Hermes 41 [1906] 142-151; METTE, H. J. : Livius und Augustus. Gymnasium 68 [1961] 269-285, repris en partie dans Wege zu Livius [Wege der Forschung 132], sous la direction de Erich Burck, Darmstadt, 1967, 158166 ; BURCK, E. : Livius und Augustus. Illinois Classical Studies 16 [1991] 269-281 ; ou SYME, R. : Livy and Augustus. Harvard Studies in Classical Philology 64 [1959] 27-87, et PETERSEN, H. : Livy and Augustus. Transactions and Proceedings of the American Philological Association 92 [1961] 440-452) et sur l'orientation politique qu'on peut attribuer à l'historien, qu'on a pu considérer aussi bien comme un républicain convaincu, opposant mal dissimulé au nouveau régime, qu'un des artisans de la restauration augustéenne. Comme travail récent, on pourra voir sur le sujet MiNÉO, B. : Tite-Live et l'histoire de Rome [Ëtudes 
reçut la citoyenneté romaine qu'en 49 av. J.-C., lorsque César, par la lex Roscia, accorda ce statut à la Gaule Cisalpine, cette partie de la péninsule dont le régime provincial ne fut aboli qu'en 42 av. J.-C., l'Italie administrative s'étendant désormais, bien au-delà de la frontière historique du Rubicon, jusqu'à la barrière naturelle des Alpes $^{7}$. À ce titre, il faisait partie de ces non Romains au sens strict sur lesquels s'était appuyé le futur Auguste, lorsqu'il était parti combattre Antoine et Cléopâtre fort du soutien que lui avaient prêté par serment les habitants de la péninsule, cette coniuratio Italiae que célèbrent les res gestae, et que, devenu le maître du monde romain, le prince avait officiellement associés au destin de l'Vrbs par la réorganisation de l'Italie en onze régions, vis-à-vis desquelles Rome n'avait désormais plus la position de domination qui avait été la sienne depuis la conquête. Il est comme son contemporain et voisin de Mantoue Virgile un des meilleurs exemples de l'intégration réussie de ce qui était resté pendant longtemps une terre de confins, assimilée par son régime de prouincia aux territoires que les légions avaient fait entrer dans l'imperium Romanum en dehors de la péninsule ${ }^{8}$.

Dans l'éloge de l'Italie que le poète mantouan avait inséré dans ses Géorgiques (2. 136-176), le thème de l'unité de la péninsule, surmontant les anciens clivages et les conflits du passé et faisant la force d'une Rome désormais sortie d'une conception étroitement urbaine de son identité, passe au premier plan ${ }^{9}$. Lorsqu'il définit ses habi-

et commentaires 107]. Paris 2006 (et L'Ab Vrbe Condita : quel instrument politique ? Cahiers des études anciennes 47 [2010] 385-408). Même si l'historien gardait sa liberté de jugement à l'égard d'Auguste (il exprimait ouvertement dans sa préface, en 9, son scepticisme envers les mesures de redressement moral prises par le prince) et restait profondément attaché aux formes traditionnelles de la res publica, que le prince s'était bien gardé de bouleverser, il n'y a pas de raison de douter de la sincérité de sa reconnaissance envers celui qui avait enfin fermé les portes du temple de Janus et institué la pax Augusta après la tourmente des gurres civiles (1. 19. 3). Il pouvait se dire l'ami du prince (Tacite souligne que son «pompéianisme » ne fit pas obstacle à cette amicitia) et il était suffisamment bien en cour pour qu'on lui confiât un rôle dans la formation du futur empereur Claude (Suétone, Vie de Claude 41 : historiam in adulescentia hortante T. Liuio... scribere adgressus est), qui, s'il n'était pas alors, loin de là, une figure de premier plan de la famille impériale, n'en était pas moins le fils de Drusus, fils de Livie.

${ }^{7}$ Nous parlons ici de l'Italie au sens administratif, non pas du concept géographique, pour lequel l'extension jusqu'aux Alpes est attesté beaucoup plus tôt, étant sensible dès la deuxième guerre punique. Sur la question de l'Italie, on pourra se reporter en dernier lieu à SIMON, M. : Le rivage grec de l'Italie romaine. La Grande Grèce dans l'historiographie augustéenne [Collection de l'École française de Rome 442]. Rome 2011, 65-125.

${ }^{8}$ Données p. ex. dans LAFFI, U. : Studi di storia romana e di diritto. Roma 2001, 245-261. Mais sur les limites de la construction d'une unité italienne sous Auguste, voir les remarques de GIARDINA, A. : L'identità incompiuta dell'Italia romana. In L'Italie d'Auguste à Dioclétien. Actes du colloque de Rome (25-28 mars 1992) [Collection de l'École Française de Rome 198]. Rome, 1994, 1-89. Pour Padoue, SARTORI, Fr. : Padova nello stato romano. Dal secolo III a.C. all'età diocleziana. In Padova antica. Da comunità paleoveneta a città romano-cristiana. Trieste 1981, 99-189, et, pour les aspects institutionnels, BASSIGNANO, M. S. : Il municipio patavino. In Padova antica 199-227.

${ }^{9}$ Sur la thématique des éloges de l'Italie et les précédents et parallèles dans la littérature latine, MARTIN, R. : Recherches sur les agronomes latins et leurs conceptions économiques et sociales. Paris 1971, 259-269. 
tants comme un genus acre uirum $(167)^{10}$, les exemples qu'il prend pour illustrer cette force humaine sont « le Ligure et les Volsques armés d'épieux » (168, Ligurem Volscosque uerutos) contre lesquels les Romains eurent à lutter à maintes reprises, les populations sabelliques (167, pubemque Sabellam), dont la composante essentielle fut celle des Samnites dont l'image est liée aux trois guerres que les légions eurent à soutenir contre eux et dont la haine que continuait à leur porter Rome trouva une illustration tragique avec le massacre des soldats de Pontius Telesinus à la Porte Colline en 82 av. J.-C. ${ }^{11}$, et en premier lieu ces Marses (167, Marsos), dont le nom était associé à celui du Marsicum Bellum, première désignation donnée à la guerre sociale au cours de laquelle Rome eut à faire face à une révolte de ses alliés qui se posaient comme la véritable Italie, dont, sur les monnaies qu'ils avaient émises, le taureau symbolique écrasait la louve qui avait nourri les jumeaux fondateurs de l' $V r b s^{12}$. Les bœufs qui sont sacrifiés à l'occasion des triomphes romains ${ }^{13}$ ne sont pas des bêtes latines, mais ombriennes, nourries dans les pâturages voisins du Clitumne (146147), issues d'une région qui ne reçut la citoyenneté romaine que par la lex Iulia de ciuitate de 90 av. J.-C. Parmi les chefs romains eux-mêmes qui sont cités aux v. 169$172^{14}$, précédant la référence à Marius, à Camille, aux Scipions et à Auguste lui-même, posé en vainqueur de l'Asie, c'est le nom des Decii qui vient en tête, renvoyant donc à cette famille chez qui le sacrifice sous forme de deuotio ducis au cours d'une bataille semble avoir été une sorte de pratique héréditaire, mais qui était d'origine non romaine, mais campanienne ${ }^{15}$. C'est donc la péninsule tout entière rangée sous la bannière de Rome et permettant à celle-ci d'y puiser ses forces que célèbre le poète :

${ }^{10}$ Virgile, Géorgiques 2. 167-169 : haec genus acre uirum, Marsos pubemque Sabellam / adsuetumque malo Ligurem Volscosque uerutos / extulit (« ce pays a produit une race d'hommes ardente, les Marses, et la jeunesse Sabellienne, et le Ligure endurci à la fatigue et les Volsques armés d'épieux »).

${ }^{11}$ Sur l'image des Samnites (et des populations sabelliques en général), voir DENCH, E. : From Barbarians to New Men. Greek, Roman and Modern Perception of Peoples from the Central Italy. Oxford 1995.

${ }^{12}$ Sur la guerre sociale, données dans HINARD, Fr. : Histoire romaine I. Des origines à Auguste. Paris 2000, 611-626, avec bibliographie p. 987. Sur les monnaies des insurgés, voir notre étude dans Le regard des autres, les origines de Rome vues par ses ennemis (début du IV siècle / début du I siècle av. J.-C.) [Annales Littéraires de l'Université de Franche-Comté 623]. Besançon-Paris 1997, 153-195.

13 Virgile, Géorgiques 2. 146-148: hinc albi, Clitumne, greges et maxima taurus / uictima, saepe tuo perfusi flumine sacro, / Romanos ad templa deum duxere triumphos (« de là, tes blancs troupeaux, Clitumne, et le taureau, la plus grande des victimes, souvent, après s'être baignés dans ton fleuve sacré, conduisirent aux temples des dieux les triomphes romains »).

${ }^{14}$ Virgile, Géorgiques 2. 169-172: haec Decios Marios magnosque Camillos, / Scipiadas duros bello et te, maxime Caesar, / qui nunc extremis Asiae iam uictor in oris / imbellem auertis Romanis arcibus Indum (« ce pays a produit les Decii, les Marius, les grands Camille, les Scipions durs à la guerre, et toi, le plus grand de tous, César, qui, déjà vainqueur aux extrêmes confins de l'Asie, repousses maintenant des citadelles romaines l'Indien désarmé »).

${ }^{15}$ Sur P. Decius Mus et ses origines campaniennes, qui reste un des seuls points solides de ce qu'on considérait naguère comme l' «État romano-capouan » que Rome était censée avoir formé au lendemain de la deditio de la cité campanienne en 443 av. J.-C., voir HEURGON, J. : Recherches sur l'histoire, la religion et la civilisation de la Capoue préromaine, des origines à la deuxième guerre punique [Bibliothèque des Écoles Françaises d'Athènes et de Rome 154]. Paris 1942, 155-342 ; HeURGON, J. : Rome et la Méditerranée occidentale jusqu'aux guerres puniques [Coll. Nouvelle Clio No. 7]. Paris 1969, 325. Sur la deuotio, Bloch R. - GuITTARD, CH. : Tite-Live, Histoire romaine, livre VIII. Paris 1987, LV-LXXXVIII. 
mais c'est une Italie qui a intégré les régions qui avaient attendu les lois de 49 et 42 av. J.-C., et donc la cité des bords du Mincio où il était né, pour que leur appartenance à cet ensemble soit officiellement reconnue. Évoquant la richesse en eaux du pays et ses lacs ${ }^{16}$, Virgile met en balance ceux des deux composantes de l'Italie, celle dont les Apennins formait la limite et celle de la plaine padane qui en est devenue part intégrante, puisqu'au Lucrin et à l'Averne de Campanie $(161,164)$ font pendant le Larius (lac de Côme, 159) et le Benacus (lac de Garde, 160), promus à l'égal de la mer Tyrrhénienne, ce mare Inferum qui, dans les représentations des Anciens, baignait le Sud de l'Italie. L'éloge de la péninsule que compose l'Italien du Nord Virgile est une célébration de l'union de toutes les parties de la tota Italia, et en particulier celles de la toute nouvelle extension que constituait la zone dont il était originaire.

Le Padouan s'accordait certainement avec le Mantouan sur le besoin de dépasser une vision trop étroitement romaine de l'Italie et il lui arrive, à lui aussi, de souligner la valeur de peuples italiens, quand bien même ils ont montré celle-ci en s'opposant à l'Vrbs : un passage du livre $\mathrm{X}$ est particulièrement significatif de ce point de vue, où il laisse percer son admiration pour les Samnites qui furent capables, des années durant, en dépit des défaites répétées et sanglantes que leur infligeaient les Romains, de s'opposer à eux et de défendre leur libertas $^{17}$. Mais ce qu'on note chez lui relève

\footnotetext{
${ }^{16}$ Virgile, Géorgiques 2. 158-164 : an mare quod supra memorem, quodque adluit infra? / anne lacus tantos? te, Lari maxime, teque, / fluctibus et fremitu adsurgens Benace marino? / an memorem portus Lucrinoque addita claustra / atque indignatum magnis stridoribus aequor, / Iulia qua ponto longe sonat unda refuso / Tyrrhenusque fretis immittitur aestus Auernis? (« rappellerai-je la mer qui la baigne au nord, et celle qui la baigne au sud ? Ou encore ses grands lacs ? Toi, Larius, le plus grand, et toi, Benacus, dressant tes flots et frémissant comme la mer? Rappellerai-je nos ports, et les digues ajoutées au Lucrin, et la mer indignée avec ses sifflements énormes aux lieux où l'onde Julienne résonne du bruit des flots qu'elle refoule au loin et où la vague Tyrrhénienne s'élance aux eaux de l'Averne ? »).

${ }^{17}$ Tite-Live 10. 31. 10-15 : Supersunt etiam nunc Samnitium bella, quae continua per quartum iam uolumen annumque sextum et quadragesimum a M. Valerio A. Cornelio consulibus, qui primi Samnio arma intulerunt, agimus; et ne tot annorum clades utriusque gentis laboresque actos nunc referam, quibus nequiuerint tamen dura illa pectora uinci, proximo anno Samnites in Sentinati agro, in Paelignis, ad Tifernum, Stellatibus campis, suis ipsi legionibus, mixti alienis, ab quattuor exercitibus, quattuor ducibus Romanis caesi fuerant; imperatorem clarissimum gentis suae amiserant; socios belli, Etruscos, Vmbros, Gallos, in eadem fortuna uidebant qua ipsi erant; nec suis nec externis uiribus iam stare poterant, tamen bello non abstinebant. Adeo ne infeliciter quidem defensae libertatis taedebat et uinci quam non temptare uictoriam malebant. Quinam sit ille quem pigeat longinquitatis bellorum scribendo legendoque quae gerentes non fatigauerunt? (« Il me reste encore à raconter de ces guerres samnites, dont le récit, sans cesse poursuivi, nous a amenés déjà à mon quatrième volume et à la quarante-sixième année de leur durée, depuis le consulat de Marcus Valerius et d'Aulus Cornelius, qui, les premiers, portèrent les armes dans le Samnium. Et, pour ne pas rappeler maintenant tant d'années de défaites subies par les deux peuples et de peines qui ne purent, cependant, vaincre ces cœurs endurcis, la dernière année dont nous avons parlé, les Samnites, sur le territoire de Sentinum, chez les Péligniens, à Tifernum, dans les plaines de Stella, avec leurs seules légions ou joints à des troupes étrangères, avaient été taillés en pièces par quatre armées, quatre généraux romains ; ils avaient perdu leur chef le plus célèbre ; leurs alliés, Étrusques, Ombriens, Gaulois, ils les voyaient dans le même état qu'eux ; ni leurs forces, ni des forces étrangères ne leur permettaient plus de rester debout; pourtant ils ne renonçaient pas à la guerre, tant la défense, même malheureuse, de leur liberté était loin de les lasser, tant ils préféraient être vaincus à ne pas tenter la victoire ! Quel est donc l'homme que rebuterait, comme écrivain ou comme lecteur, la longueur de ces guerres, qui ne lassèrent pas ceux qui les faisaient ?»).
} 
surtout d'un souci, au fond plus banal, de mettre en valeur sa petite patrie padouane et de la faire figurer en bonne place dans son œuvre. La patauinitas joue aussi dans sa vision historique et Rome ne lui fait pas oublier Padoue.

De cette place attribuée à sa petite ville padane, on a même un trait éclatant, sinon paradoxal : dans une œuvre qui est destinée à relater le passé glorieux de Rome, on commence par rencontrer sinon à proprement parler Padoue, du moins une Troia euganéenne, qui apparaît comme l'ancêtre de la ville natale de l'historien, et ces $A b$ Vrbe condita libri s'ouvrent sur un exposé non sur les origines des Latins et donc la fondation de l'Vrbs ou d'une des antiques métropoles latines dont elle était considérée comme issue, Lavinium ou Albe, mais sur celles du peuple chez qui l'historien était né, dans la lointaine Vénétie ${ }^{18}$. Il relate en effet, avant d'en venir à l'histoire d'Énée (et par l'effet d'une composition en chiasme, vu que, lorsqu'il avait donné les noms des deux héros dans sa première phrase, il avait mentionné Énée avant Anténor), comment le peuple auquel appartenait sa ville natale doit son origine au Troyen Anténor $^{19}$. Cette allusion, en fait, à l'origine de Padoue, en une position aussi excep-

${ }^{18}$ Tite-Live 1. 1-4 : Iam primum omnium satis constat Troia capta in ceteris saeuitum esse Troianos, duobus, Aeneae Antenorique, et uetusti iure hospitii et quia pacis reddendaeque Helenae semper auctores fuerant, omne ius belli Achiuos abstinuisse. Casibus deinde uariis Antenorem cum mutitudine Enetum, qui seditione ex Paphlagonia pulsi et sedes et ducem rege Pylaemene ad Troiam amisso quaerebant, uenisse in intimum maris Hadriatici sinum, Euganeisque qui inter mare Alpesque incolebant pulsis Enetos Troianosque eas tenuisse terras. Et in quem primo egressi sunt locum Troia uocatur pagoque inde Troiano nomen est, gens uniuersa Veneti appellati. Aeneam ab simili clade domo profugum, sed ad maiora rerum initia ducentibus fatis, primo in Macedoniam uenisse, inde in Siciliam quaerentem sedes delatum, ab Sicilia classe ad Laurentem agrum tenuisse. Troia et huic loco nomen est ("Voici tout d'abord un fait généralement admis : après la prise de Troie tous les Troyens furent traités avec la dernière rigueur. Cependant, en ce qui concerne Énée et Anténor, en respect du droit d'une ancienne hospitalité et parce qu'ils avaient constamment recommandé de faire la paix et de rendre Hélène, les Grecs ne les soumirent pas au droit de la guerre. Après des aventures diverses, Anténor pénétra jusqu'à l'extrémité du golfe Adriatique avec une troupe nombreuse d'Hénètes qui, chassés de Paphlagonie par une sédition et ayant perdu devant Troie leur roi Pylémène, cherchaient un lieu où s'établir et un chef. Les Euganéens qui habitaient entre la mer et les Alpes furent chassés de leur pays et celui-ci fut occupé par les Hénètes et les Troyens. Le lieu du débarquement s'appelle Troie et le village qui s'y forma par la suite porte le nom de bourg troyen. L'ensemble du peuple reçut le nom de Vénètes. Énée, forcé de quitter sa patrie à la suite du même désastre, était appelé par les destins à fonder une plus grande puissance. Il se rendit d'abord en Macédoine. De là, toujours en quête d'un lieu où s'établir, il passa en Sicile. De Sicile, il arriva avec ses navires dans le territoire des Laurentes. Cet endroit porte, lui aussi, le nom de Troie »).

${ }^{19}$ Le souvenir de la fondation de Padoue par Anténor était maintenu par la célébration, tous les trente ans, des ludi cetasti, connus pour la participation du Padouan Paetus Thrasea à l'époque de Néron, qui fut une des raisons qui poussèrent le prince à l'obliger au suicide (Tacite, Annales 16. 21 . 1 : ad postremum Nero uirtutem ipsam excindere concupiuit interfecto Thrasea Barea Sorano, olim utrisque infensus et accedentibus causis in Thraseam, quod senatu egressus est cum de Agrippina referretur, ut memoraui, quodque Iunenalium ludicro parum spectabilem operam praebuerat; eaque offensio altius penetrabat, quia idem Thrasea Pataui, unde ortus erat, ludis cetastis a Troiano Antenore institutis habitu tragico cecinerat. Néron voulut à la fin exterminer la vertu même, par la mort de Paetus Thraseas et Barea Soranus. Tous deux, il les haïssait depuis longtemps, mais des raisons supplémentaires jouaient dans le cas de Thraseas : il était sorti du Sénat, comme je l'ai rappelé, pendant la délibération qui suivit la mort d'Agrippine et aux jeux des Juvenalia, il n'avait pas fait voir un zèle assez empressé ; cette offense était d'autant plus sensible à Néron, qu'à Padoue, d'où il était issu, le même Thraseas, lors des ludi cetasti institués par le

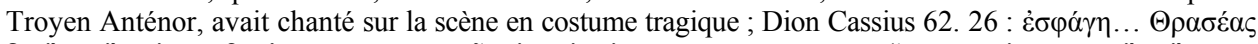

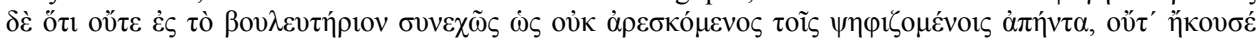


tionnelle dans l'œuvre, tient bien évidemment à ce qu'on pourrait considérer comme un heureux hasard : les Vénètes, comme les Latins, pouvaient se prévaloir d'une légende de fondation troyenne et le conditor de la ville padane Anténor pouvait être ainsi mis en parallèle avec le fils d'Anchise à qui Rome était rattachée, au moins indirectement. Mais il faut bien avouer qu'on ne s'attend guère à voir ces non Latins figurer en si bonne place dans une histoire de Rome : à titre de comparaison, il n'en est fait nulle mention chez Denys d'Halicarnasse dans le récit parallèle des Antiquités romaines. Sans doute l'historien grec, dans son désir de présenter l'Vrbs non seulement comme une ville grecque, mais même comme la seule ville authentiquement hellénique dans une péninsule barbare (et en utilisant dans sa démonstration non seulement des traditions faisant intervenir des Grecs véritables, comme Évandre et ses Arcadiens ou les compagnons grecs d'Héraklès, mais également les Troyens d'Énée, non distingués des Hellènes dont ils avaient pourtant été les adversaires ${ }^{20}$ ), avait-il des raisons particulières pour passer sous silence l'existence d'une tradition qui risquait de lui sembler entrer en concurrence avec celle regardant le Latium. Mais, en ce qui concerne l'historien padouan, on peut au moins estimer que ces Livres depuis la fondation de Rome commencent ainsi par un hors-sujet et que cette référence à Anténor et ses Hénètes n'apporte rien à la narration, d'autant plus que Tite-Live aurait pu tout aussi bien évoquer une tradition comme celle qui faisait des Élymes de Sicile eux aussi des Troyens ${ }^{21}$. C'est bien évidemment le patriotisme local qui l'a poussé à mentionner cette autre légende d'origine troyenne ${ }^{22}$, à rappeler ainsi que sa cité natale elle aussi, tout aussi bien que l'Vrbs qui dominait l'oikouménè, était née de Troie. Sa volonté de présenter les Vénètes sous un jour favorable est d'autant plus sensible qu'il se garde

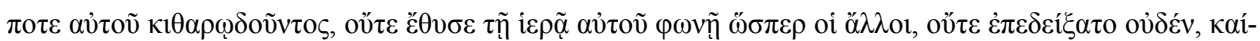

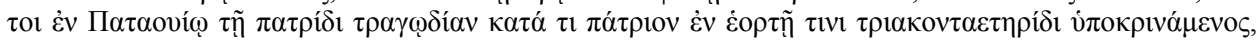
quant à Thraseas, il (mourut) parce qu'il ne venait pas assidûment au Sénat, n'approuvant pas les décrets qui s'y rendaient ; parce qu'il n'avait jamais écouté Néron chanter sur la lyre ; parce qu'il n'avait jamais sacrifié, comme les autres, à sa divine voix ; parce qu'il n'avait donné aucun spectacle, bien qu'à Padoue, sa patrie, il eût, suivant une coutume nationale, fait représenter une tragédie dans une fête qui se célébrait tous les trente ans). Sur les ludi cetasti, JACOBS, L. D. : Ludi cetasti Patavinorum. Athenaeum 67 (1989) 275-281 ; LINDERSKI, J. : Games in Patavium. Ktêma 17 (1992) 55-67. Dans l'exposé livien, on notera que le nom de Padoue ne figure pas, il est question seulement de l'origine du peuple vénète ; mais à ce stade de l'exposé non plus, pour le Latium, le nom des métropoles successives, Lavinium, Albe, et bien sûr surtout Rome, autour de laquelle se concrétiseront les destins qui sont évoqués, n'est mentionné.

${ }^{20}$ Pour le sens de la démarche de Denys d'Halicarnasse dans son livre I, on se reportera à MUSTI, D. : Tendenze nella storiografia romana e greca su Roma arcaica. Studi su Livio e Dionigi d'Alicarnasso [Quaderni Urbinati di Cultura Classica 10]. Roma 1970; GABBA, E. : Dionysius and the History of Archaic Rome [Sather Classical Lectures 56]. Berkeley 1991, Fromentin, V. : Denys d'Halicarnasse, Antiquités Romaines, Introduction générale - Livre 1. Paris 1998, 8-42. Sur la signification de la désignation comme « ville grecque » d'une ville non grecque comme Rome, FRASCHETTI, A. : Eraclide Pontico e Roma città greca. In CASSIO, A. C. - Musti, D. (ed.) : Tra Sicila e Magna Grecia. Aspetti di interazione culturale nel secolo IV a.C. Atti del convegno, Napoli 19-20 marzo 1987. Pisa 1991 (= AION 11), 81-95; VANOTt, G. : Roma polis hellenis, Roma polis tyrrhenis. Riflessioni sul tema. Mélanges de l'École Française de Rome (Antiquité) 111 (1999) 217-255.

${ }^{21}$ L'origine troyenne des Élymes est évoquée dans Thucydide 6. 2. 3, Apollodore, cité dans Strabon 6. 1. 3 (254), Denys d'Halicarnasse 1. 52. 1-3, Virgile, Énéide 5. 35-41, Plutarque, Vie de Nicias 1. 3.

${ }^{22}$ Pour l'ensemble des données, on pourra se reporter à BRACCESI, L. : La leggenda di Antenore. Da Troia a Padova. Padova 1984. 
bien d'évoquer une version peu flatteuse pour ses compatriotes de la légende d'Anténor, où celui-ci, loin d'être présenté comme un modèle de justice qui aurait souhaité faire droit aux justes réclamations de Ménélas, l'était comme un traître qui avait lié sa ville aux envahisseurs achéens. Cette interprétation malveillante, à laquelle Denys d'Halicarnasse fait une allusion rapide lorsqu'il avance, en 1, 46, 1, parmi les raisons possibles de la chute de Troie, «la trahison des Anténorides »" ${ }^{23}$, bien sûr n'apparaît pas chez Tite-Live, alors que certains historiens avaient dû la mettre en avant pour rejeter sur le fondateur de la cité vénète une accusation qui avait été portée, notamment dans une optique anti-romaine, sur Énée lui-même. Tout comme le pius Aeneas, le héros troyen à qui les Vénètes rattachaient leur histoire se devait d'être irréprochable. L'orgueil local ne l'aurait pas supporté.

Le même orgueil local se retrouve dans la fière remarque, en 5.33. 9, que, lorsque les Étrusques étendirent leur domination sur la zone padane, y établissant une dodécapole à l'image de celle qu'ils formaient en Toscane, l'« angle des Vénètes » échappa à cette mainmise, le peuple d'Anténor étant le seul à avoir gardé son indépendance ${ }^{24}$. Et il est encore plus sensible dans le long excursus du chapitre 2 du livre $\mathrm{X}$ où TiteLive, allant contre le principe qu'il avait posé comme une règle fondamentale de sa manière d'écrire l'histoire au livre précédent ${ }^{25}$, abandonne la narration des faits qui

\footnotetext{
${ }^{23}$ Nous avons étudié cette question dans Dionigi di Alicarnasso, o le ragioni di un silenzio. In ZAMPIERI, G. (dir.) : Padova per Antenore. Atti della giornata di studio tenutasi il 14 dicembre 1989 presso il Museo Civico Archeologico agli Eremitani [Studi Padovani 5]. Padova 1990, 125-135. Sur le thème de la trahison d'Anténor et son lien avec la question de la trahison d'Énée, BRACCESI : La leggenda (n. 22)126-146. Anténor est présenté comme un traître par Lykophron, Alexandra 340-343, Cornelius Sisenna, cité par Servius, commentaire à Virgile, Énéide 1. 242 (= fr. $1 H R R, 1$ Chassignet), Alexandre d'Éphèse, cité dans l'Origo Gentis Romanae 9. 1, un Lutatius (pour lequel on hésite entre l'affranchi Lutatius Daphnis, de la fin de l'époque républicaine, ou Q. Lutatius Catulus, le collègue de Marius au consulat en 102 av. J.-C., qui remporta avec lui la bataille de Verceil contre les Cimbres et qui périt victime des proscriptions), qui lui adjoint Énée (Origo Gentis Romanae 9. 2). Le thème de la trahison d'Énée, fondé sur l'hostilité posée dès l'Iliade, en 13. 765-787, entre Priam et le fils d'Anchise, est attesté sans doute au IV ${ }^{\mathrm{e}}$ siècle av. J.-C., avec Ménécrate de Xanthos, cité par Denys d'Halicarnasse 1. 48.3 (= FGrHist 769 F 3) ; également Servius, commentaire à Virgile, Énéide 1. 488, 647, scholie de Vérone à Virgile Énéide 2. 217. Sur ce point, UsSANI, V. : Enea traditore. Studi Italiani di Filologia Classica 22 (1947) 109-123. Sur l'affirmation fautive de Servius, suivant lequel Tite-Live aurait considéré aussi bien Anténor qu'Énée comme des traîtres (commentaire à Virgile, Énéide, 1, 242: hi enim prodidisse dicuntur secundum Liuium), voir CAPDEVILLE, G. : Antenore e Diomede, rivali letterari e ideologici di Enea, à paraître.

${ }^{24}$ Tite-Live 5. 33. 9 : Ei in utrumque mare uergentes incoluere urbibus duodenis terras, prius cis Appenninum ad inferum mare, postea trans Appenninum totidem, quot capita originis erant, coloniis missis, quae trans Padum omnia loca, - excepto Venetorum angulo qui sinum circumcolunt maris, - usque ad Alpes tenuere (« Maîtres du territoire qui s'étend de l'une à l'autre mer, les Étrusques y bâtirent douze villes et s'établirent d'abord en deçà de l'Apennin vers la mer Inférieure ; ensuite de ces villes principales originelles furent expédiées autant de colonies qui, à l'exception de l'angle des Vénètes, qui sont établis sur le pourtour du golfe, occupèrent tout le pays au-delà du Pô jusqu'aux Alpes »).

${ }^{25}$ Tite-Live 9. 17. 1 : Nihil minus quaesitum a principio huius operis uideri potest quam ut plus iusto ab rerum ordine declinarem uarietatibusque distinguendo opere et legentibus uelut deuerticula amoena et requiem animo meo quaererem (« On peut voir qu'il n'y a rien que j'ai davantage cherché à éviter depuis le début de cet ouvrage que de m'écarter de la suite des événements plus qu'il n'était légitime et, en coupant mon œuvre par des digressions, de chercher à offrir à la fois à mes lecteurs comme
} 
regardent l'histoire de Rome pour décrire par le détail ce qui est alors advenu dans son pays natal, lorsque Cléonyme, quittant le Sud de l'Italie où Tarente l'avait appelé à son secours, se lança dans une expédition au fond de l'Adriatique, qui échoua piteusement devant la résistance des Padouans ${ }^{26}$. Ici, assurément, l'historien s' « écarte

d'agréables promenades et à mon esprit un repos »). On remarquera cependant que l'historien posait ce principe au moment même où allait y déroger, en traitant sur trois chapitres de ce qui serait advenu à Alexandre s'il avait décidé de tourner ses armes contre les Romains, dans le fameux excursus sur le conquérant macédonien.

${ }^{26}$ Tite-Live 10. 2. 1-15 : Eodem anno classis Graecorum Cleonymo duce Lacedaemonio ad Italiae litora adpulsa Thurias urbem in Sallentinis cepit. Aduersus hunc hostem consul Aemilius missus proelio uno fugatum compulit in naues; Thuriae redditae ueteri cultori Sallentinoque agro pax parta. Iunium Bubulcum dictatorem missum in Sallentinos in quibusdam annalibus inuenio et Cleonymum, priusquam confligendum esset cum Romanis, Italia excessisse. Circumuectus inde Brundisii promunturium medioque sinu Hadriatico uentis latus, cum laeua importuosa Italiae litora, dextra Illyrii Liburnique et Histri, gentes ferae et magna ex parte latrociniis maritimis infames, terrerent, penitus ad litora Venetorum peruenit. Expositis paucis qui loca explorarent, cum audisset tenue praetentum litus esse, quod transgressis stagna ab tergo sint inrigua aestibus maritimis, agros haud procul campestres cerni, ulteriora colles uideri; esse ostium fluminis praealti quo circumagi naues in stationem tutam possint - Meduacus amnis erat-, eo inuectam classem subire flumine aduerso iussit. Grauissimas nauium non pertulit alueus fluminis; in leuiora nauigia transgressa multitudo armatorum ad frequentes agros tribus maritimis Patauinorum uicis colentibus eam oram peruenit. Ibi egressi praesidio leui nauibus relicto uicos expugnant, inflammant tecta, hominum pecudumque praedas agunt et dulcedine praedandi longius usque a nauibus procedunt. Haec ubi Patauium sunt nuntiata - semper autem eos in armis accolae Galli habebant - in duas partes iuuentutem diuidunt. Altera in regionem qua effusa populatio nuntiabatur, altera, ne cui praedonum obuia fieret, altero itinere ad stationem nauium - milia autem quattuordecim ab oppido aberatducta. In naues ignaris custodibus interemptis impetus factus territique nautae coguntur naues in alteram ripam amnis traicere. Et in terra prosperum aeque in palatos praedatores proelium fuerat refugientibusque ad stationem Graecis Veneti obsistunt; ita in medio circumuenti hostes caesique: pars capti classem indicant regemque Cleonymum tria milia abesse. Inde captiuis proximo uico in custodiam datis pars fluuiatiles naues, ad superanda uada stagnorum apte planis alueis fabricatas, pars captiua nauigia armatis complent profectique ad classem immobiles naues et loca ignota plus quam hostem timentes circumuadunt; fugientesque in altum acrius quam repugnantes usque ad ostium amnis persecuti captis quibusdam incensisque nauibus hostium, quas trepidatio in uada intulerat, uictores reuertuntur. Cleonymus uix quinta parte nauium incolumi, nulla regione maris Hadriatici prospere adita discessit. Rostra nauium spoliaque Laconum, in aede Iunonis ueteri fixa, multi supersunt qui uiderunt Pataui. Monumentum naualis pugnae eo die quo pugnatum est quotannis sollemni certamine nauium in oppidi medio exercetur (« La même année, une flotte grecque, que commandait le Lacédémonien Cléonyme, ayant abordé en Italie, prit la ville de Thuriae, chez les Sallentins. Envoyé contre ces ennemis, le consul Aemilius n'eut besoin que d'une bataille pour les mettre en fuite et les rejeter dans leurs navires. Thuriae fut rendue à ses anciens habitants et la paix acquise au territoire sallentin. Ce fut Junius Bubulcus, dictateur, qui fut envoyé chez les Sallentins, à ce que je vois dans certaines annales, et Cléonyme, avant d'être obligé de lutter avec les Romains, quitta l'Italie. De là, tournant le cap de Brindes et emporté par les vents au milieu du golfe Adriatique, comme, à gauche, la côte sans ports de l'Italie, à droite les Illyriens, les Liburnes et les Istriens, nations sauvages et décriées, en grande partie pour leur piraterie, l'effrayaient, il s'enfonça dans le golfe et arriva à la côte vénète. Il y débarqua quelques hommes pour reconnaître les lieux ; et, ayant appris que la côte qui s'étendait devant lui n'était qu'une bande de terre, qu'en la traversant, on trouvait derrière des lagunes entretenues par les marées, que, non loin, on distinguait des champs, d'abord en plaine, qu'au delà, on voyait des collines, qu'il y avait là l'embouchure d'un fleuve très profond, par où les éclaireurs avaient vu des navires conduits par un détour à un mouillage sûr (c'était le Meduacus), Cléonyme ordonna à sa flotte de s'y diriger et de remonter ce fleuve. Mais les navires les plus lourds n'y trouvèrent pas des fonds suffisants. Passée sur des bâtiments plus légers, une foule de soldats parvint à des territoires peuplés, trois bourgades maritimes des Padouans occupant cette côte. Là les Grecs débarquent, en laissant quelques hommes pour garder les bateaux, prennent d'assaut les villages, brûlent les 
de la suite des événements », puisque le devenir ultérieur du chef lacédémonien, une fois qu'il a été chassé de la péninsule sallentine soit par peur de la menace représentée par l'arrivée de l'armée romaine et de son chef le dictateur Junius Bubulcus, soit, dans la version alternative présentée dans le texte livien, après une défaite que celle-ci lui aurait infligée, sous les ordres de M. Aemilius Paullus qui était un des consuls de cette année 302 av. J.-C. ${ }^{27}$, ne concernait plus du tout Rome. Si Tite-Live relate cette expédition du prince spartiate contre les Vénètes et s'étend aussi complaisamment sur un événement qui ne fut qu'un raid momentané et sans lendemain, c'est bien évidemment parce que cela lui donne l'occasion de faire apprécier à ses lecteurs romains un haut fait de ses compatriotes, que ceux-ci ignoraient probablement, mais dont les Padouans estimaient pouvoir tirer une gloire légitime. Pendant longtemps, les éperons pris aux navires ennemis - en un pendant vénète des rostres enlevés en 338 av. J.-C. aux pirates antiates qui décoraient la tribune aux harangues de l' $V r b s-$ avaient orné le temple de leur Junon locale. Et des jeux nautiques annuels, toujours vivants à l'époque où l'historien écrivait, rappelait, au jour anniversaire de la bataille, cette victoire sur le chef lacédémonien : elle était en quelque sorte pour eux l'équivalent des grandes victoires navales romaines, celles remportées à Myles par le consul Caius

maisons, emmènent hommes et troupeaux comme butin et, dans la joie du pillage, s'avancent trop loin de leurs navires. À cette nouvelle, à Padoue, où le voisinage des Gaulois tenait toujours les habitants sous les armes, on divise la jeunesse en deux corps : l'un est mené vers la région que les ennemis dispersés ravageaient, disait-on ; l'autre, pour ne pas rencontrer de pillards, va par une route opposée vers le mouillage des bateaux grecs, qui était à quatorze milles de la ville. On attaque ces bateaux, où on tue quelques gardes, et les matelots terrifiés sont forcés de les amener à l'autre rive. Sur terre, on n'est pas moins heureux en combattant les pillards dispersés ; aux Grecs qui fuient vers leur mouillage, les Vénètes barrent le chemin ; ainsi ces ennemis sont cernés et taillés en pièces ; certains, faits prisonniers, révèlent que la flotte et le roi Cléonyme sont à trois milles de là. Alors, laissant les prisonniers en garde au village le plus proche, les Padouans, les uns sur des embarcations fluviales, bien faites, avec leur fond plat, pour passer là où il y a peu d'eau, les autres sur les bâtiments enlevés aux Grecs, tous remplis d'hommes en armes, se dirigent vers la flotte grecque et attaquent de tous côtés ses vaisseaux immobiles, qui craignent ces parages inconnus plus que l'ennemi; et quand ils s'empressent de fuir vers la mer, plutôt que de repousser l'attaque, les Padouans les poursuivent jusqu'à l'embouchure du fleuve, prennent et brûlent certains d'entre eux, qui, dans leur hâte, se sont jetés sur des hauts fonds, et reviennent vainqueurs. Cléonyme, sauvant à peine un cinquième de ses navires et n'ayant abordé avec succès aucun point de la mer Adriatique, s'en éloigna. Les éperons des navires et les dépouilles des Laconiens furent accrochés dans le vieux temple de Junon ; il reste encore beaucoup de gens qui les ont vus à Padoue. Le souvenir de ce combat naval, en son jour anniversaire, chaque année, est célébré par une joute solennelle de bateaux sur le fleuve, au milieu de la ville »). L'expédition de Cléonyme a fait l'objet d'une étude de Larenzo BRACCESI, L. : L'avventura di Cleonimo, a Venezia prima di Venezia [Saggi materiali universitari 13, Serie di antichità e tradizione classica 12]. Padova 1990.

${ }^{27}$ La chronologie de l'année 302 av. J.-C., telle que la présente le récit livien, concentre tous les événements liés à l'expédition de Cléonyme sur cette année. Elle est incompatible avec la présentation des faits chez Diodore de Sicile, qui fait état pour 303 d'une guerre entre les Romains (alliés aux Lucaniens) et Tarente (20.104. 1), place l'arrivée de Cléonyme en Italie cette année-là (2), lui attribue un accord avec les Lucaniens puis avec Métaponte contre laquelle il s'est tourné (3-4), avant de repartir pour Corcyre, d'où il reviendra bientôt (20. 105. 1), menant quelques opérations avec des résultats mitigés (1-2), avant de retourner à Corcyre et de se désintéresser des lors des affaires italiennes (3). Sur les problèmes posés par la divergence entre les deux auteurs, voir OAKLEY, ST. P. : A Commentary on Livy, Books VI-X. Volume IV, Book X. Oxford 2005, 48-52 ; Grelle, Fr. - Silvestrini, M. : La Puglia nel mondo romano. Storia du una periferia. Dalle guerre sannitiche alla guerra sociale. Bari 2013, 45-48. 
Duilius sur les Carthaginois en 260 av. J.-C. au cours de la première guerre punique - qui avait été marquée par l'érection des colonnes rostrales - et par Octave à Nauloque, près du cap Pélore, à Actium contre Antoine et Cléopâtre, et à Myles - qui donnaient lieu à des célébrations sous forme de naumachies encore à l'époque d'Ausone $^{28}$. De ce fait, nous glanons de nombreuses informations sur la cité natale de l'historien, qui nous fait connaître ce culte de Junon et ces jeux nautiques, fait une description topographique précise de la région, avec son paysage de bras fluviaux et de lagunes, son habitat avec des hameaux indépendants, décrit des realia comme le type d'embarcation adapté au peu de profondeur des eaux, laisse entrevoir l'organisation militaire de la cité, avec une iuuentus rapidement mobilisable et capable de répondre aux directives de ses chefs. On sent, au-delà de l'orgueil bien compréhensible du Padouan fier d'exposer les exploits de ses ancêtres, le plaisir qu'il prend à parler de sa petite patrie et à la faire découvrir à un public romain sans doute surpris de rencontrer une telle évocation d'une petite ville provinciale - qu'il aurait certainement plutôt eu tendance à considérer de haut, à se gausser de sa patauinitas à la manière d'Asinius Pollion - dans une œuvre consacrée aux res gestae du peuple-roi.

Mais il serait réducteur de ne considérer ces pages où l'historien padouan évoque sa cité que sous l'angle du campanilisme et de la gloriole locale. Il faut prendre au sérieux ce qu'il dit quand il débute son œuvre par une allusion à la légende d'Anténor. Nulle différence n'est posée dans la phrase initiale entre Énée et Anténor, cités ensemble et présentés comme soumis au même destin. Et, comme ce qui advient au fondateur de la Troia vénète puis de Padoue est relaté d'abord, c'est en quelque sorte lui qui sert de modèle à Énée, dont on commence par dire qu'il a connu un sort analogue à celui d'Anténor. Certes, Tite-Live ne pousse pas l'outrecuidance jusqu'à affirmer que le fatum de sa ville natale était identique à celui de l'Vrbs, il est bien dit du fils d'Anchise qu'il « était appelé par les destins à fonder une plus grande puissance » ( $a d$ maiora rerum initia ducentibus fatis, 1.1.4), il va jusqu'à taire le nom de Padoue, ce qui est une manière de ne pas la poser expressément comme une autre Rome, égale à elle, mais il n'en reste pas moins que Rome n'est plus un cas unique, puisque la petite cité vénète, dont la fondation est au moins suggérée, lui est associée, partage une communauté de destin. Padoue devient en fait une autre Rome et les éléments que l'historien évoque à son propos, quand bien même il ne le précise pas, viennent renforcer cette homologie des deux patries de Tite-Live. Anténor est comme Énée un modèle de pietas et l'allusion à l'hospitium ne manque pas de rappeler au lecteur qu'il avait accordé l'hospitalité dans sa maison à Ménélas au cours de l'ambassade où il avait tenté de régler pacifiquement le conflit, qu'il avait protégé son hôte contre les menées de Pâris et que, comme le fils d'Anchise qui ne s'engagea dans la guerre que contre son gré, il était de ceux qui tenaient avant tout à la paix. Ce à quoi il donne naissance en terre vénète, un peuple nouveau formé de la fusion de deux éléments, les Hénètes et les Troyens, n'est pas sans analogie avec le peuple latin constitué par

${ }^{28}$ Ausone, Idylle 15. 208-219; le poète évoque des jeux nautiques liés à la victoire d'Actium dans la mer en face de Cumes, à celle de Myles sur le lac Averne, à celle de Nauloque, au large du cap Pélore en Sicile. Horace faisait déjà allusion à une reproduction de la bataille d'Actium, mais organisée par Lollius dans un cadre privé, dans Épitres 1. 18. 61-64. 
Énée à partir des Aborigènes et des Troyens, le roi de l'élément non-troyen, que ce soit Pyléménè ou Latinus, disparaissant pour laisser la place au seul chef troyen et, inversement, le nom de Troyens ne subsistant que comme désignation d'un lieu-dit limité. Ce nouveau peuple, dans les deux cas, se forme par la victoire sur l'ennemi qui s'y oppose, les Euganéens dans un cas, les Rutules dans l'autre, et sa formation est marquée par la fondation d'une ville nouvelle, Padoue en Vénétie et dans le Latium Lavinium, en attendant les autres métropoles que seront Albe et surtout Rome. Bref il existe une parenté profonde entre les Vénètes d'Anténor et les Latins d'Énée et l'entrée dans la cité romaine de 49 av. J.-C. n'a fait qu'officialiser ce qui était une communauté de destin posée ab origine entre la ville établie sur les bords du Bacchiglione et l' $V r b s^{29}$.

C'est dans cette optique d'une Padoue qui est à l'image de Rome qu'il faut lire l'excursus du livre X. Pas plus que, contrairement à ce que la formulation de l'historien laisserait entendre, l'excursus sur Alexandre du livre IX ne rompt la logique d'un livre centré sur la reprise inattendue de Rome au lendemain de la terrible défaite qu'a été la capitulation des Fourches Caudines ${ }^{30}$, l'excursus sur l'expédition manquée de Cléonyme en pays vénète ne doit être tenu pour une digression gratuite, un moment de relâche où l'historien abandonnerait Rome pour se plonger dans le souvenir nostalgique et complaisant de sa petite patrie du Nord. Car, là encore, Padoue est indissociable de Rome et c'est la cité des bords du Tibre qui transparaît derrière ce qui est dit de la ville de Vénétie. Jusque dans des détails comme celui des rostres pris à l'ennemi et exposés par les vainqueurs, ou de la sorte de naumachie qui conserve la mémoire de la victoire, une cité répond à l'autre. Comme les Romains, les Padouans sont des citoyens-soldats, des paysans qui vaquent à leurs occupations agricoles ou pastorales mais, dès que le besoin se fait sentir, se muent en guerriers valeureux. Leur courage s'appuie sur le sens de la discipline, sur une organisation qui relève de la scientia militaris : la manœuvre qui conduit à leur succès contre l'envahisseur grec ne le cède en rien à ce qu'un chef romain pouvait demander à ses légions et obtenir d'elles. C'est

${ }^{29}$ Ainsi une suggénéia existe entre Rome et Padoue, pour reprendre ce terme dont on connait l'importance pour les relations entre cités et peuples dans le monde antique. Sur cette notion, MUSTI, D. : Sull'idea di suggeneia in iscrizioni greche. Annali della Scuola Normale Superiore di Pisa 32 (1963) 225-239 ; CURTY, O. : Les parentés légendaires entre cités grecques. Catalogue raisonné des inscriptions contenant le terme suggéneia et analyse critique [Hautes Études du Monde Gréco-Romain 20]. Genève 1995.

${ }^{30}$ Le livre IX débute, dès la première phrase, par l'évocation de cette terrible défaite. Mais celleci, traitée assez rapidement au début du livre, fait place à la description de la remontée progressive de l'Vrbs qui, à en croire l'historien (et contre la vérité historique), aurait été capable, l'année même qui suivit celle du désastre, de triompher de l'ennemi samnite qui venait de la vaincre. Ce triomphe est étendu à la démonstration de la supériorité de Rome sur tout ennemi, quel qu'il fut, avec la démonstration de ce qui aurait été, inévitablement, la déroute face à elle de celui qui passait pour le plus grand chef militaire de tous les temps, Alexandre. Sur cette articulation profonde du livre IX, voir nos remarques dans Aspects politiques et aspects militaires dans le livre IX de Tite-Live : les figures de L. Papirius Cursor et de Q. Fabius Maximus Rullianus. In Devillers, O. - MeYers, J. (dir.) : Pouvoir des hommes, pouvoir des mots, des Gracques à Trajan. Hommages au Professeur Paul-Marius Martin. Louvain-Paris 2009, 331-349, et À propos de l'excursus Alexandri de Tite-Live : les chefs romains potentiellement vainqueurs d'Alexandre dans le livre IX des Histoires. Boulletine de l'Association Guillaume Budé, 2015, 143-172. 
d'ailleurs, précise Tite-Live, la nécessité de se prémunir contre la menace incessante des Gaulois qui garantit la qualité militaire des Padouans : un lecteur romain ne peut manquer de songer à ce que représenta pour sa ville le tumultus Gallicus, le danger que constitua pendant des décennies le retour d'une attaque de la part des bandes celtiques du genre de celle qui, avec Brennus, avait manqué d'emporter à jamais la cité - et qui lui avait au moins infligé la honte de la seule pénétration ennemie qu'elle ait connue sur le sol sacré délimité par le pomerium. Il faut peut-être même pousser plus loin ici la liaison entre les deux cités. Sans doute, dans son récit des événements de 390 av. J.-C., Tite-Live ne parle-t-il pas des Vénètes. Mais on sait que, pour Polybe, la raison qui avait poussé les Gaulois vainqueurs à abandonner la ville dont ils s'étaient emparés est que, au loin dans le Nord, les Vénètes avaient attaqué leur territoire, forçant Brennus et les siens à s'en retourner chez eux (2.2.18) ${ }^{31}$. Il y a toutes chances que Tite-Live ait connu cette tradition : la mention des Gaulois, dans ce passage du livre $\mathrm{X}$, peut au moins y faire penser - et rappeler que les Vénètes pouvaient ainsi être considérés comme ceux qui avaient sauvé Rome, et cela peut-être à plus juste titre qu'un Camille dont il est inutile de rappeler combien le développement de la légende (dont Polybe ne parlait pas) est tardif et artificiel. En tout cas, citer l'ennemi celte, à propos de Padoue, renvoyait, pour des Romains, au souvenir de toutes ces guerres gauloises dans lesquelles les Vénètes avaient été leurs alliés - comme lors de la grande invasion de 228 av. J.-C., où, comme le précise Polybe, ils avaient conclu immédiatement une alliance avec les ambassadeurs envoyés par le Sénat (2. 2. 23) ${ }^{32}$.

Ce ne sont pas cependant ces souvenirs des guerres gauloises qui sont les plus importants pour comprendre la valeur de cet excursus du livre X. Cette fois, l'ennemi qu'affrontent les Padouans n'est pas celtique, mais grec. Et c'est ici aussi un ennemi commun aux Vénètes et aux Romains : avant de raconter les déboires de Cléonyme contre ses compatriotes, Tite-Live avait évoqué, assez rapidement et sous deux formes alternatives, les rapports hostiles qui avaient opposé les troupes de l'Vrbs au chef lacédémonien, soit que celui-ci eût subi une défaite au cours d'une bataille, soit qu'il eût préféré fuir plutôt que d'avoir à affronter un tel danger (10. 2. 2-3). Cette fois encore, comme en 390 av. J.-C., les Vénètes se comportent donc en alliés objectifs des Romains, appuient l'action qu'ils ont eux-mêmes entreprise contre ce qui peut apparaître comme un identique adversaire.

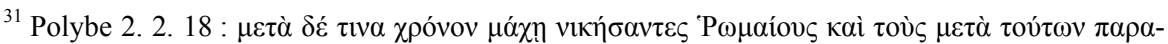

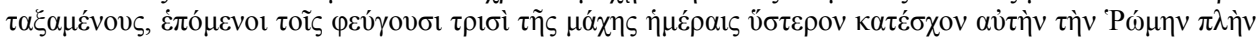

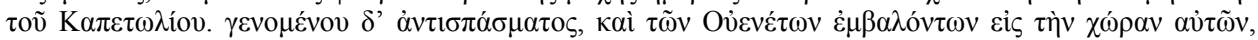

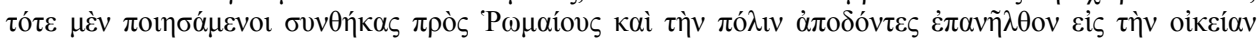
(«quelque temps après, les Gaulois défirent en bataille rangée les Romains et leurs alliés, se mirent aux trousses des fuyards et, trois jours après, s'emparèrent de la ville même de Rome, à l'exception du Capitole. Mais, au même moment, une attaque des Vénètes contre leur territoire fit diversion et ils rentrèrent chez eux après avoir traité avec les Romains et leur avoir rendu la ville »).

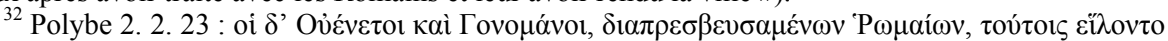
$\sigma 0 \mu \mu \alpha \chi \varepsilon \tilde{i v}$ ( « les Vénètes et les Cénomans s'entendirent avec les ambassadeurs envoyés par les Romains et conclurent une alliance avec eux »). Pour Tite-Live, on peut signaler que la seule occurrence du nom de la Vénétie en dehors des passages que nous étudions les met en rapport avec les Gaulois : il s'agit de la mention, en 39. 22. 6, pour 186 av. J.-C., au fait que les Gaulois s'établirent sur le territoire sur le site de la future Aquilée. 
Mais il faut surtout tenir compte de ce que représente Cléonyme, dont la personnalité et le rôle historique ouvrent des perspectives tout autres que l'ennemi gaulois. Il est un des généraux étrangers à qui Tarente fit successivement appel pour la défendre contre la menace représentée par les populations indigènes, Iapyges, Lucaniens et bientôt Romains. Cléonyme, avec son intervention des années 303/302 av. J.-C. au secours de la grande cité jadis fondée par Sparte, s'inscrit dans la longue série des chefs mercenaires à qui les Tarentins s'en remirent dans leurs luttes extérieures, après le roi de Sparte Archidamos, en 344/338 av. J.-C., le souverain épirote Alexandre le Molosse, de 336 à 331 av. J.-C., Agathocle de Syracuse qui mit ses talents militaires au service de Tarente entre 323 et 317 av. J.-C. et précède le dernier et le plus célèbre d'entre eux, Pyrrhos, dont l'expédition italienne débuta en 280 et s'acheva par son départ en 275 av. J.-C. ${ }^{33}$. Dans ces conditions, derrière Cléonyme, dont la présence en Italie fut évanescente et les heurts avec les Romains, s'ils ont une réalité historique, durent en tout état de cause rester très limités, ne pouvait être senti par les Romains que comme l'antécédent de celui qui avait été pour eux un adversaire d'une tout autre portée, le roi d'Épire, émule d'Alexandre, dont l'Vrbs eut tant de mal à triompher et qui avait été le premier souverain du monde grec tel que l'établissement de l'empire macédonien l'avait mis en place que les légions avaient affronté et vaincu. La figure de Cléonyme, tout secondaire qu'il fût, était indissociable de celle du vainqueur d'Ausculum en 279 av. J.-C., enfin vaincu à Bénévent en 275 av. J.-C.

Bien sûr l'existence du « trou » de Tite-Live et le fait que la guerre contre Pyrrhos était traitée par Tite-Live dans sa deuxième décade qui ne nous est pas parvenue ont pour conséquence que nous ignorons la manière dont l'historien padouan relatait les faits, que les sèches énumérations des periochae des livres XII-XIV ne nous permettent pas de réellement saisir ${ }^{34}$. Mais il n'y a nul doute que c'était à ses yeux un des moments cruciaux de l'histoire de Rome, celui où, avec la prise de Tarente, s'achevait la conquête de l'Italie péninsulaire et où s'entrevoyait déjà l'étape suivante, le conflit avec Carthage qui allait s'ouvrir dès que l'Vrbs franchirait le détroit de Messine et s'impliquerait dans les affaires de Sicile. En outre, par son importance en tant que souverain hellénistique, et qui plus est émule d'Alexandre et considéré comme le second après lui dans la liste des plus grands chefs militaires que le monde avait connus ${ }^{35}$,

${ }^{33}$ Sur ces chefs étrangers au service de Tarente, données dans WUILLEUMIER, P. : Tarente, des origines à la conquête romaine [Bibliothèque des Écoles Françaises d'Athènes et de Rome 148]. Paris 1939, 77-81 (Archidamos), 81-88 (Alexandre le Molosse), 91, 96-98 (Agathocle), 94-96 (Cléonyme), 99-137 (Pyrrhos) ; LÉVÊQUE, P. : Pyrrhos [Bibliothèque des Écoles Françaises d'Athènes et de Rome 185]. Paris 1957, 395-550; ou Grelle, Fr. - Silvestrini, M. : La Puglia nel mondo romano. Bari 2013, 34-37 (Archidamos), 37-43 (Alexandre le Molosse), 45-48 (Agathocle), 48-50 (Agathocle), 94-104 (Pyrrhus).

${ }^{34}$ Les témoignages des auteurs se rapportant à la période qui nous concerne ici ont été commodément rassemblés dans TORELLI, M. R. : Rerum Romanarum fontes ab anno CCXCII ad annum CCLXV a. Ch. N. collegit atque notis illustravit Marina R. Torelli [Biblioteca degli Studi Classici ed Orientali 14g. Pisa 1978.

${ }^{35}$ Dans l'épisode de la rencontre entre Hannibal et Scipion au cours de l'ambassade (fictive) que celui-ci aurait menée à Éphèse en 192 av. J.-C. est dressée la liste de ceux qui étaient considérés comme les meilleurs généraux dans le monde hellénistique d'alors : Pyrrhus y figure en deuxième place, après Alexandre et avant Hannibal (Tite-Live, se référant à Claudius Quadrigarius et à Acilius, 35. 14. 5-12= Acilius, frg. 5 HRR, 7 Chassignet, Claudius Quadrigarius, frg. 64 A HRR, 65 Chassignet). 
Pyrrhus occupait une place particulière, annonçant la future domination de Rome sur le monde méditerranéen, y compris dans sa partie orientale, grecque. Si on lit donc ce que Tite-Live relate dans ce chapitre du livre $\mathrm{X}$ de l'échec subi par Cléonyme devant Padoue en songeant à celui que l'Vrbs eut à affronter quelques années plus tard, le parallélisme est patent : le destin échu au précédent chef grec appelé à la défense de Tarente annonce la défaite du roi d'Épire, lui aussi contraint à une fuite sans gloire devant ces indigènes italiens qu'il avait cru pouvoir vaincre sans coup férir. Ce qu'ont fait les Padouans en 302 av. J.-C. annonce ce que feront les Romains vis-à-vis de Pyrrhos, en quelque sorte ils montrent à Rome le modèle à suivre.

La cité d'Anténor préfigure ainsi ce qu'il adviendra bientôt à l' $V r b s$ et c'est là certainement la raison profonde de l'insistance de Tite-Live sur cet épisode, qui reste malgré tout secondaire. Mais il ne faudrait pas non plus forcer la note et lui donner une importance qu'il n'a pas : il a sans doute cette valeur d'exemplum, mais l'historien ne va pas jusqu'à inverser le rapport entre ses deux patries et attribuer à sa ville natale un rôle plus grand que celui qui fut le sien dans l'histoire. Déjà, même si ce qui s'est passé à Padoue est traité avec bien plus de précision que les événements survenus dans la péninsule sallentine qui ont précédé, on peut tout aussi bien dire que la cité d'Anténor n'a fait que reproduire ce que les Romains venaient eux-mêmes de réaliser face à Cléonyme dans la région de Tarente. Et le raid lancé par le chef spartiate contre le pays vénète n'a évidemment pas l'ampleur de la guerre de Pyrrhus. TiteLive garde le sens des proportions et, s'il tient à souligner le rôle de sa petite patrie, il n'oublie pas, comme il le disait à propos d'Anténor par rapport à Énée en un passage que nous avons déjà cité, que le second «était appelé par les destins à fonder une plus grande puissance » (1. 1. 4). Padoue, cité troyenne comme Rome et donc sœur de l'Vrbs, restait néanmoins, y compris pour Tite-Live, dans l'ombre de la ville des bords du Tibre.

Le caractère subordonné de Padoue par rapport à Rome apparait clairement dans le dernier passage de l'œuvre livienne - du moins dans ce qui nous est parvenu - où il est question de la ville d'Anténor. Il s'agit cette fois d'un court passage du livre XLI, se rapportant à des faits survenus en 174 av. J.-C. (41.27. 3-4) :

Consules uotis in Capitolio nuncupatis in prouincias profecti sunt. Ex iis M. Aemilio senatus negotium dedit, ut Patauinorum in Venetia seditionem conprimeret, quos certamine factionum ad intestinum bellum exarsisse et ipsorum legati attulerant. Legati, qui in Aetoliam ad similis motus conprimendos ierant, renuntiarunt coerceri rabiem gentis non posse. Patauinis saluti fuit aduentus consulis; neque aliud, quod ageret in prouincia, cum habuisset, Romam redit

Les consuls, après le prononcé des voeux au Capitole, partirent pour leurs provinces. L'un d'eux, M. Aemilius, fut chargé par le Sénat de mettre fin, en Vénétie, à une sédition des Padouans, chez qui la lutte de partis avait allumé la guerre civile, ainsi que l'avait également annoncé une délégation qu'ils avaient envoyée. (4) Des délégués, envoyés en Étolie pour 
réprimer de semblables mouvements, écrivirent qu'il était impossible de mettre fin à la rage de ce peuple. Les Padouans furent sauvés par l'arrivée du consul ; celui-ci, n'ayant rien d'autre à faire dans sa province, revint à Rome.

Cette fois, on ne peut pas dire que l'image de la ville natale de l'auteur des $A b$ Vrbe condita libri soit très favorable. Même si, à la différence de ce qui est dit dans le même contexte des Étoliens, l'historien ne taxe pas ses compatriotes de rage, rabies, ils n'en sont pas moins en proie aux déchirements internes, à une situation d'oppositions politiques, de lutte entre les factions, qui débouche sur ce que le texte désigne comme une véritable guerre civile, intestinum bellum. D'eux-mêmes, ils sont bien incapables de trouver une solution : ils sont obligés de s'en remettre à Rome, à qui ils envoient une ambassade pour la prier de remettre de l'ordre dans leurs affaires. Ce que fait la puissance romaine, dont c'est le consul Aemilius en personne qui mène à bien cette mission : c'est de l'Vrbs, non plus de ses propres ressources que la cité vénète reçoit la solution à ses maux internes. Tite-Live emploie le mot saluti, qui est un terme très fort : pour les Padouans, les Romains sont de véritables sauveurs. On est aux antipodes de ce qui avait pu sembler être le cas au moment de l'attaque de Brennus contre Rome, où, si on se réfère à la présentation polybienne des faits (2. 2. 18), c'est en quelque sorte l'attaque lancée par les Vénètes contre les Gaulois qui avait sauvé l'Vrbs, ou même en 302 av. J.-C. vis-à-vis de l'intervention de Cléonyme en Italie.

Qualifier ainsi les Romains de sauveurs ne doit pas être ramené à la rhétorique complaisante qui se développait dans le monde grec, magnifiant les habitants de l'Vrbs au point d'en faire des sauveurs du monde entier. Dans le cas précis de Padoue, les citoyens durent effectivement ressentir que, dans une situation critique de déchirements internes où leur cité leur semblait devoir sombrer, Rome était venue leur apporter le salut. Des données extérieures à Tite-Live en apportent la preuve. La ville a en effet livré onze inscriptions qui témoignent de l'existence d'un système de datation local, exprimé par les lettres NA suivies d'un chiffre. William V. Harris a proposé d'interpréter ces lettres comme l'abréviation de n(ostro) a (nno), Jerzy Linderski comme celle de na(tali die) : le point important est qu'il s'agit là d'indications temporelles, référées à une ère propre à Padoue. Or le point de départ de celle-ci, autant qu'on puisse en juger par les chiffres qui sont donnés, paraît être 173 av. J.-C. (soit $581 a b$ Vrbe condita), ce qui renvoie aux événements narrés par Tite-Live au livre $\mathrm{XLI}^{36}$. Cela signifie que la remise en ordre de la cité, opérée par l'intervention de Rome, est apparue comme un nouveau départ, une véritable refondation de celle-ci : l'antique fondation d'Anténor n'existait pour ainsi dire plus que grâce à Rome et à travers l'opération salvatrice qui avait été mise en œuvre par l'autre cité troyenne.

Dans ces circonstances, ce n'est bien sûr plus Padoue qui peut passer pour un modèle pour les Romains, la rapport est inversé et c'est l'Vrbs qui est obligée d'intervenir dans les affaires de sa petite sœur pour y rétablir une situation compromise. Mais,

${ }^{36}$ HARris, W. V. : The era of Patavium. Zeitschrift für Papyrologie und Epigraphik 27 (1977) 283-293 ; LINDERSKI, J. : Natalis Patavii. Zeitschrift für Papyrologie und Epigraphik 50 (1983) 227232, LIU, J. : The era of Patavium again. Zeitschrift für Papyrologie und Epigraphik 162 (2007) 281-289. 
la valeur d'exemple pouvant jouer dans les deux sens, on peut dire que cette fois l'exemplum donné par la ville vénète est négatif : il montre ce qu'il ne faut pas faire et, étant donné les rapports de consanguinité entre Vénètes et Romains, le danger qui s'est concrétisé à Padoue rappelle aux habitants de l'Vrbs ce qu'ils doivent faire pour ne pas connaître un tel état de discorde. Les Padouans en sont arrivés à ce que l'historien n'hésite pas à qualifier de bellum intestinum, leurs luttes politiques, les tensions partisanes de leurs factiones mettent la cité dans une situation sans issue, dont ils sont incapables de sortir par eux-mêmes. Tite-Live ne nous précise pas quelles sont ces factions aux prises entre elles, il n'évoque même pas, comme il l'a fait à propos de la ville étrusque d'Arezzo, également en proie aux déchirements internes, une hostilité entre la plèbe et l'aristocratie locale ${ }^{37}$. Mais le peu qu'il en dit suffit : cela montre que sa cité natale n'a pas su faire régner la concordia, que les conflits politiques ou sociaux, dont la réalité est indéniable et dont on ne saurait nier qu'ils sont un des moteurs de l'histoire, le cas de Rome dont une bonne partie de l'histoire a été dominée par le conflit des ordres le prouve amplement, n'ont pas su trouver une solution de nécessaire compromis - comme l'Vrbs a su le faire, notamment par la concession des droits aux plébéiens qui leur permettait de demeurer dans la cité et de participer à la grandeur de son destin. Au fond, l'exemple de Padoue montre ce qui aurait pu advenir à Rome, mais que celle-ci a su heureusement surmonter.

Ce dernier passage montre bien que l'amour de sa petite patrie n'obnubile pas le Padouan Tite-Live au point d'en donner une image unilatérale, et unilatéralement favorable dans son œuvre. Certes il lui reste attaché, est heureux de rappeler son histoire et, lorsque cela est possible, de magnifier les hauts faits de ses compatriotes. Mais il est loin d'en faire un modèle et n'hésite pas, comme pour les événements de 174 av. J.-C., à l'ériger en contre-modèle. Cependant, que ce soit dans un sens positif ou dans un sens négatif, on peut dire que Padoue est toujours pensée par rapport à Rome, l'autre cité troyenne, celle, bien plus que sa petite cité vénète, dont le sort était de devenir la nouvelle Troie qui était appelée par les dieux à destin grandiose. Ce que l'historien natif de Padoue rédige est bien sûr une histoire de Rome, non un ouvrage centré sur sa ville d'origine comme ce De origine Patauina que mentionne l'Origo Gentis Romanae ${ }^{38}$. Que Tite-Live soit issu de la cité d'Anténor ne l'empêche évidemment pas de se sentir avant tout comme un Romain, d'être préoccupé avant tout de

${ }^{37}$ Dans le cas d'Arezzo, Tite-Live en 10. 5.13 (cf. 10. 3. 2) emploie le terme de plebs pour désigner les éléments populaires en révolte contre la famille des Cilnii qui dominait la cité. Sur la situation sociale en Étrurie et les problèmes que pose la définition des termes employés par les auteurs anciens, MAZZARINO, S. : Sociologia del mondo etrusco e problemi della tarda etruscità. Historia 6 (1957) 98122 ; FRANKFORT, TH. : Les classes serviles en Étrurie. Latomus 18 (1959) 3-22 ; HEURGON, J. : Les pénestes étrusques chez Denys d'Halicarnasse (IX, 5, 4). Latomus 18 (1959) 715-723 (mais contra BENELLI, E. : Sui cosidetti penesti etruschi. Parola del Passato 51 [1996] 335-344); HeURGON, J. : La vie quotidienne chez les Étrusques. Paris 1961, 57-94 ; TORELLI, M. : Per una storia dello schiavismo in Etruria. Dialoghi di Archeologia 8 (1974-1975) 67-78 ; TORELl, M. : Storia degli Etruschi. Roma-Bari 1981, 199-214, 215-255.

${ }^{38}$ Voir Origo Gentis Romanae 1. 6. Sur cet ouvrage, voir les remarques de RICHARD, J.-C. (éd.) : Pseudo-Aurélius Victor, Les origines du peuple romain. Paris 1983, 111-112, n. 14. 
l'Vrbs et de sa place de puissance dominatrice du monde, telle qu'elle s'est réalisée à travers les vicissitudes de l'histoire, avec ses péripéties contrastées, ses succès et ses revers, les actions de ses summi uiri et de ceux qui ont joué un rôle négatif. Dans cette vaste perspective, sa cité natale n'occupe assurément qu'une place marginale et il ne cherche pas à lui attribuer davantage que ce qu'il n'en a été réellement. Mais elle n'en joue pas moins dans sa construction historique une fonction utile de contrepoint vis-à-vis de Rome, son souvenir n'est pas totalement absent et se révèle parfois significatif - dans des épisodes aussi différents que la victoire contre Cléonyme en 302 av. J.-C. derrière lequel on devine en filigrane la figure de Pyrrhus, ou le bellum intestinum de 174 av. J.-C., où Padoue faillit sombrer dans la discordia. Tite-Live fournit un bon exemple de ce que pouvait être pour un Romain non issu de l'Vrbs l'appartenance à ses deux patries, la grande patrie romaine, mais également la petite patrie qui l'avait vu naître ${ }^{39}$. Il fournit donc un bon exemple aussi de la réussite de la politique augustéenne, d'intégration de l'Italie dans l'ensemble romain, par-delà la mémoire des luttes et des tensions du passé.

Dominique Briquel

Université de Paris-Sorbonne

1 rue Victor Cousin, 75230 Paris Cedex 05

dominique.briquel@gmail.com

${ }^{39}$ Cette question a fait l'objet du travail de Bonjour, M. : Terre natale. Études sur une composante affective du patriotisme romain. Paris 1975. 